\title{
Human Brucellosis: Methods of Diagnosis and Risk Factors among Egyptian Patients at Assiut Fever Hospital
}

\author{
Essam A. El-Moselhy', Tahseen S. Soliman', Ayman A. Abd El-Rahman', \\ Diaa M. El-Tiby', Hesham H. Amin', Bahaa M. Badr \\ ${ }^{I}$ Community Medicine Department, Faculty of Medicine, Al-Azhar University, Assiut, \\ Egypt \\ Original \\ Article \\ ${ }^{2}$ Community Medicine Department, Faculty of Medicine, Al-Azhar University, Cairo, Egypt \\ ${ }^{3}$ Internal Medicine Department, Faculty of Medicine, Al-Azhar University, Assiut, Egypt \\ ${ }^{4}$ Tropical Medicine Department, Faculty of Medicine, Al-Azhar University, Cairo, Egypt, \\ ${ }^{5}$ Clinical Pathology Department, Faculty of Medicine, Al-Azhar University, Assiut, Egypt, \\ ${ }^{6}$ Medical Microbiology and Immunology Department, Faculty of Medicine, Al-Azhar \\ University, Assiut, Egypt
}

\begin{abstract}
Background: Human brucellosis, a common zoonotic disease, is major public health problem in many countries worldwide including Egypt.

Objectives: To define brucellosis patients' risk-factors and to assess diagnostic lab methods of brucellosis at Assiut Fever Hospital.

Patients and Methods: The study recruited 98 patients with brucellosis and an equal number of controls. All participants were subjected to interview, clinical examination, and lab investigations.

Results: Older age, males, rural residence, low socioeconomic status were significant risk-factors (OR=3.76, 2.04, 2.86, 2.72; respectively). Occupations had animals' contact were significant risk-factor $(\mathrm{OR}=4.7)$; the most risky were butchers/ slaughter workers $(\mathrm{OR}=8.0)$ and farmers/dairy workers $(\mathrm{OR}=3.59)$. Longer occupational exposure was risk-factor $(\mathrm{OR}=15.57)$. The main significant presenting symptoms were fever and musucloskeletal affections. The main significant signs were high temperature and hepato- and spleno-megaly. Standard agglutination test (SAT) titer 1/320 was the cut-off point for diagnosis and significantly lies in area under the ROC curve, sensitivity $=96.4 \%$ and specificity $=100.0 \%$. Blood culture was positive in $58.2 \%$ of cases with no significant differences between SAT titer and blood culture positivity. ELISA IgM and IgG results were positive in $69.4 \%$ and $65.3 \%$ of the cases with no significant differences between SAT titer and IgM and IgG results.

Conclusions: Human brucellosis has many preventable risk-factors; its diagnosis depends mainly on presence of riskfactors, clinically suspected, and SAT titer $\geq 1 / 320$.
\end{abstract}

Key Words: Brucellosis, clinical, diagnosis, risk-factors, sociodemographic.

Received: 12 September 2019, Accepted: 18 September 2019

Corresponding Author: Essam A. El-Moselhy; MD; Department of Community Medicine, Faculty of Medicine, AlAzhar University, Assiut, Egypt, Tel.: +20 10063170, E-mail: dr.elmoselhy@azhar.edu.eg - dr_elmoselhy@yahoo.com

ISSN: 2090-7265, Journal of Recent Advances in Medicine Vol 1 issue 1 January 2020

\section{INTRODUCTION}

Human brucellosis is a common neglected, re-emerging, zoonotic disease with worldwide distribution; it jeopardizes human health and animal production $^{[1]}$. It's caused by bacteria of genus Brucella; human pathogens are B. abortus, melitensis, suis, etc. [2]. The disease is infectious; transmitted to humans by contact with fluids of infected animals or derived food products ${ }^{[3]}$. Over than 500.000 cases are reported yearly in many countries ${ }^{[4]}$. Brucellosis prevalence had increased in many developing areas ${ }^{[5]}$; up-to $17.0^{\%} \%^{[6]}$. However, its epidemiology had drastically changed over the past decade because of socioeconomic, sanitary, global travel development, and political reasons ${ }^{[7]}$. As an effect of farm animal screening and vaccination programs, and pasteurization of dairy-products, the overall incidence of brucellosis become lower ${ }^{[8]}$. 
In Egypt, brucellosis still endemic; its true incidence is underestimated ${ }^{[9]}$. In rural Gharbia, brucellosis seroprevalence was $1.7 \%^{[10]}$. While, brucellosis seroprevalence among exposed workers in Sharkia was $21.0 \%{ }^{[11]}$. A hospital-based study in AinShams University Hospitals showed brucellosis was the commonest infectious disease, in adults, causes fever of unknown origin (FUO) ${ }^{[12]}$. Also, $3.0 \%$ and $11.0 \%$ of 10,130 acute febrile illness (AFI) patients, from 13 Egyptian Fever Hospitals, were positive for brucella using culture and serology, respectively[13].

Brucellosis is systemic infection; any body organ can be involved ${ }^{[2,14-14]}$. It has high morbidity for humans and animals; it's an important cause of public health problem and economic loss in many developing countries $^{[15]}$. It's included in the differential diagnosis of FUO/AFI in endemic areas. It's a disease of protean manifestations; however fever is fixed. Examination is non-specific; but lymphadenopathy, hepatomegaly, and/or splenomegaly are often present ${ }^{[16]}$. Acute illness is characterized by high swinging fever, rigors, sweating, lethargy, headache, and joint/musclepains $^{[17]}$.

Development of definitive diagnostic test for brucellosis is an elusive target ${ }^{[16]}$. Various serological tests have been deployed for brucellosis screening in humans ${ }^{[18]}$. Definitive and dependable method for brucella diagnosis depends on its isolation from blood or other tissues ${ }^{[19]}$. Because Brucella is difficult to culture, diagnosis usually depends on positive Brucella agglutination or enzyme linked immunosorbent assay (ELISA) test results with high titers of antibody $(\mathrm{Ab})^{[14]}$. Serological methods have proven useful in the study of brucellosis in developing countries because they are simple, cost effective, robust and reproducible ${ }^{[20]}$

\section{OBJECTIVES OF THE STUDY}

They are to determine the sociodemographic, lifestyle, and risk-factors of brucellosis patients; to define duration of antibiotic use and relapse rate; to evaluate diagnostic lab methods (standard agglutination test (SAT), blood culture, ELISA)) of brucellosis; and to assess SAT as a significant, standard diagnostic lab method for brucellosis.

\section{PATIENTS AND METHODS}

I. Study design, setting, and time: A hospital-based, case-control, follow-up study design was chosen to perform this research at Assiut Fever Hospital, from February 2018 to January 2019.

II. Administrative design: Approvals to conduct the study were obtained.

III. Study population: Patients with clinical and epidemiological features suspected of brucellosis, admitted to the hospital to verify the diagnosis, were the target population.
IV. Patients and controls: Patients were checked by SAT to prove the diagnosis, titer $\geq 1320 /$ was considered positive (case). Equal number of apparently healthy subjects (other out-patients without abnormal findings) was enrolled as controls.

V. Ethical consideration: Study protocol was approved by local Ethical Committee of Al-Azhar Faculty of Medicine, Assiut. Study aims were explained to the participants; accordingly informed consents were taken from them.

\section{Study tools:}

1. Interviewing form: A specially designed, comprehensive interviewing form was used. Socioeconomic level was determined according to El-Gilany et al. ${ }^{[21]}$ with modification.

2. Clinical examination: The participants were subjected to full clinical examinations.

3. Investigations: The needed investigations (e.g. pelvic-abdominal sonography, CT-abdomen, etc.) were done for the cases.

\section{Laboratory tools and methods:}

4.1. Routine laboratory tools: The participants (patients and controls) were subjected to complete blood count $(\mathrm{CBC})$, erythrocyte sedimentation rate (ESR), liver- [alanine amino-transferase (ALT), aspartate amino-transferase (AST), total serum bilirubin] and renal-functions (urea and creatinine).

4.2. Specific laboratory tools and methods: Whole blood samples were collected in $5 \mathrm{ml}$ plain Vacutainer tubes and transported directly to the laboratory where they left to clot, then centrifuged for 15 minute at speed of $1500 \mathrm{~g}$, finally sera were separated and preserved at $-20^{\circ} \mathrm{C}$ until tested.

4.2.1. SAT: Serum samples, from the participants, were analyzed using suspension of B. abortus and melitensis (Wellcome Laboratories, UK). The procedure was according to Salata ${ }^{[22]}$. Agglutinins detected in serum are usually IgM or IgG. In some sera, a blocking factor may interfere with agglutination at low serum dilution; may be due to presence of $\operatorname{IgA}$ or other non-agglutinating $\mathrm{Ab}$. Positive results, available after $24 \mathrm{hrs}$, were defined as any sample showing visible agglutination with naked eye after gentle agitation of the mixture. Any positive subject of the controls was excluded.

4.2.2. ELISA (for the patients only): ELISA is based on reaction of Abs in the sample tested with Ag adsorbed on a polystyrene surface. Unbound immune globulin is washed-off and an enzyme labeled with anti-human globulin binds the Ag-Ab complex in a second step. After a new washing step, bound conjugate is developed with the aid of a substrate solution (tetramethylbenzidine) to render a blue 
colored soluble product that turns into yellow after adding acid stopping solution ${ }^{[23]}$.

Results interpretation: $\mathrm{Ab}$-index=Sample optical density (OD)/cut-off serum mean OD x 10; Ab-index $<9$ : -ve, 911-: equivocal, and $>11$ : +ve. Samples with equivocal results must be retested and/or a new sample obtained for confirmation. Samples with the Abindex $<9$ were considered as not having IgG specific Abs against Brucella. Samples with Ab-index $>11$ were considered as having IgG specific Abs against Brucella $^{[23]}$.

4.3. Blood culture (for the patients only): The most conclusive mean of proving the diagnosis of brucellosis is a positive culture ${ }^{[4]}$. Blood samples were inoculated aseptically into blood culture bottles containing serum dextrose broth agar and subculture done every three days. The medium $\mathrm{pH}$ was adjusted in between 6.6 and 7.4; sterilized using autoclave at $121^{\circ} \mathrm{C}$ for 20 minutes with $1 \%$ glucose and $5 \%$ inactivated serum-horse before dispensing into Petri dish or tubes for slants.

5. Follow-up: Patients were followed-up for 3 months to monitor duration of treatment and relapse by SAT, ELISA, and/or blood culture as required.

\section{VII-Statistical analysis}

Data analysis was done using statistical package for the social sciences version20. Data were presented as mean \pm standard deviation (SD) for quantitative variables and frequency and percentage for qualitative variables. Groups' comparison was done using independent sample t-test for quantitative data and Yates chi-square $(\chi 2)$ or Fischer's exact (FE) tests, as appropriate, for qualitative variables. To determine risk-factors, odds ratio (OR) was used. Receiver operated characteristic (ROC) curve was constructed with area under curve (AUC). It provides a useful way to evaluate the sensitivity and specificity for quantitative diagnostic tool that categorize cases into one of two groups. Analysis was done to detect the cut-off point of SAT titer to detect patients with brucellosis. $P$-value $<0.05$ was considered statistically significant difference for $\mathrm{t}-, \chi^{2}$, and FE tests. While, $95 \%$ confidence interval (CI) or exact confidence limits (ECL) were used, as appropriate, for OR.

\section{RESULTS}

Among 150 cases suspected clinically to have brucellosis, diagnosis was proved in $98(65.3 \%)$ cases by SAT; B. abortus (38.8\%), melitensis $(18.4 \%)$, and mixed (42.8\%). The $\mathrm{M} \pm \mathrm{SD}$ of hospital stay, antibiotics' courses duration, and time for relapse occurrence were $13.0457 .68 \pm 29.94,8.15 \pm 26.67,4.52 \pm$ day; respectively (Table 1$)$.

Table 1: Frequency distribution of the studied cases according to clinical characteristics

\begin{tabular}{|c|c|c|}
\hline Variables & Number $=98$ & Percent \\
\hline \multicolumn{3}{|l|}{ Cases suspected to have brucellosis $(n=150)$ : } \\
\hline Cases proved by laboratory diagnosis to haven't brucellosis & $52 / 150$ & 34.7 \\
\hline Cases proved by laboratory diagnosis to have brucellosis & $98 / 150$ & 65.3 \\
\hline Brucella abortus & 38 & 38.8 \\
\hline Brucella melitensis & 18 & 18.4 \\
\hline Mixed infection & 42 & 42.8 \\
\hline \multicolumn{3}{|l|}{ Seasonal variation: } \\
\hline Winter (December-January- February) & 12 & 12.2 \\
\hline Spring (Marsh-April-May) & 20 & 20.4 \\
\hline Summer (June-July-August) & 38 & 38.8 \\
\hline Autumn (September- October-November) & 28 & 28.6 \\
\hline Hospital stay (Mean \pm SD* day) & \multicolumn{2}{|c|}{$13.04 \pm 4.52$} \\
\hline Duration of complete symptoms disappearance (Mean \pm SD day) & \multicolumn{2}{|c|}{$7.15 \pm 2.31$} \\
\hline Total time of antibiotic use in- and out-hospital (Mean \pm SD day) & \multicolumn{2}{|c|}{$26.67 \pm 8.15$} \\
\hline Time of relapse occurring (Mean \pm SD day) & \multicolumn{2}{|c|}{$29.94 \pm 57.68$} \\
\hline Relapse rate: & 26 & 26.5 \\
\hline
\end{tabular}

*SD: Standard deviation

Age group 4563-year, males, and rural residence are significant risk-factors for brucellosis $(\mathrm{OR}=3.76$, 2.04, 2.86; respectively). Unskilled labor $[\mathrm{OR}=2.17]$ and low socioeconomic level $(\mathrm{OR}=2.72)$ are significant risk-factors. Drinking un-pasteurized, rawmilk, and slaughtering animals 1 month before disease are significant risk-factors $(\mathrm{OR}=2.63,3.64,4.42$; respectively) (Table2). 
Table 2: Distribution of the studied cases with brucellosis and controls according to their demographic, socioeconomic, lifestyle, and clinical risk-factors

\begin{tabular}{|c|c|c|c|c|c|}
\hline \multirow{2}{*}{ Variables } & \multicolumn{2}{|c|}{ Cases $(n=98)$} & \multicolumn{2}{|c|}{ Controls $(n=98)$} & \multirow{2}{*}{$\begin{array}{c}\text { OR }(95 \% \mathrm{CI})^{*} \\
\mathrm{OR}(95 \% \mathrm{ECL})^{* *}\end{array}$} \\
\hline & No. & $\%$ & No. & $\%$ & \\
\hline \multicolumn{6}{|c|}{ Demographic risk-factors } \\
\hline \multicolumn{6}{|l|}{ Age (years): } \\
\hline $3-18$ & 14 & 14.3 & 18 & 18.4 & $0.74(0.32-1.69)^{*}$ \\
\hline $19-44$ & 62 & 63.3 & 73 & 73.5 & $0.59(0.31-1.14)^{*}$ \\
\hline $45-63$ & 22 & 22.4 & 7 & 7.1 & $3.76(1.43-10.3)^{*}$ \\
\hline \multicolumn{6}{|l|}{ Gender: } \\
\hline Male & 64 & 65.3 & 46 & 46.9 & $2.04(1.11-3.77)^{*}$ \\
\hline Female & 34 & 34.7 & 52 & 53.1 & $0.49(0.27-0.9)^{*}$ \\
\hline \multicolumn{6}{|l|}{ Residence: } \\
\hline Rural & 65 & 66.3 & 40 & 40.8 & $2.86(1.53-5.33)^{*}$ \\
\hline Urban & 33 & 33.7 & 58 & 59.2 & $0.35(0.19-0.65)^{*}$ \\
\hline \multicolumn{6}{|c|}{ Socioecono $\neg$ mic risk-factors } \\
\hline \multicolumn{6}{|l|}{ Educational status: } \\
\hline Illiterate & 57 & 58.2 & 39 & 39.8 & $2.1(1.14-3.88)^{*}$ \\
\hline Elementary & 17 & 17.3 & 25 & 25.5 & $0.61(0.29-1.29)^{*}$ \\
\hline Secondary & 14 & 14.3 & 15 & 15.3 & $0.92(0.39-2.17)^{*}$ \\
\hline University & 10 & 10.2 & 19 & 19.4 & $0.47(0.19-1.15)^{*}$ \\
\hline \multicolumn{6}{|l|}{ Occupational status: } \\
\hline House wife & 30 & 30.6 & 37 & 37.8 & $0.73(0.38-1.37)^{*}$ \\
\hline Unskilled labor & 43 & 43.9 & 26 & 26.5 & $2.17(1.14-4.13)^{*}$ \\
\hline Skilled labor & 17 & 17.3 & 23 & 23.5 & $068(0.32-1.46)^{*}$ \\
\hline Professional & 8 & 8.2 & 12 & 12.2 & $0.64(0.22-1.78)^{*}$ \\
\hline \multicolumn{6}{|l|}{ Socioeconomic level: } \\
\hline Low & 63 & 64.3 & 39 & 39.8 & $2.72(1.47-5.07)^{*}$ \\
\hline Middle & 27 & 27.6 & 38 & 38.8 & $0.6(0.31-1.14)^{*}$ \\
\hline High & 8 & 8.2 & 21 & 21.4 & $0.33(0.12-0.83)^{*}$ \\
\hline \multicolumn{6}{|c|}{ Lifestyle risk-factors } \\
\hline Eating cottage-cheese (unprocessed) & 56 & 57.1 & 38 & 38.8 & $2.11(1.14-3.88)^{*}$ \\
\hline Drinking un-pasteurized milk & 49 & 50.0 & 27 & 27.6 & $2.63(1.39-4.98)^{*}$ \\
\hline Drinking raw-milk & 33 & 33.7 & 12 & 12.2 & $3.64(1.65-8.12)^{*}$ \\
\hline Eating ice-cream from street vendor & 21 & 21.4 & 8 & 8.2 & $3.07(1.2-8.04)^{*}$ \\
\hline Breeding animals at home & 27 & 27.6 & 11 & 11.2 & $3.01(1.32-6.98)^{*}$ \\
\hline Slaughtering animals 1 month before disease onset & 12 & 12.2 & 3 & 3.1 & $4.42(1.13-25.04)^{* * *}$ \\
\hline Follow preventive measures at dealing with risk & 6 & 6.1 & 11 & 11.2 & $0.52(0.15-1.61)^{* * *}$ \\
\hline \multicolumn{6}{|c|}{ Clinical risk-factors } \\
\hline Past history of similar attack & 43 & 43.9 & 6 & 6.1 & $11.99(4.62-36.28)^{* *}$ \\
\hline Family history of similar attack & 17 & 17.3 & 4 & 4.1 & $4.93(1.51-20.81)^{* *}$ \\
\hline History of diseases (e.g. DM, liver \& renal disease, etc) & 49 & 50.0 & 38 & 38.8 & $1.58(0.86-2.9)^{*}$ \\
\hline
\end{tabular}

*CI: Confidence interval

**CI: Exact confidence limits 
Occupation that has animal contact is significant risk-factor for brucellosis $(\mathrm{OR}=4.7)$. The significant occupations risk-factors are butchers/slaughtering $(\mathrm{OR}=8.0)$ and farmers/dairy workers $(\mathrm{OR}=3.59)$. Exposure $\geq 20$ year has the highest significant risk $(\mathrm{OR}=15.57)$ (Table 3).

All symptoms and signs are significantly common among cases than controls except jaundice, hypertension/heart disease, and tender spine (Table 4). Mean $\mathrm{Hb}$ level and RBCs count are significantly lower among cases. Meanwhile, means of 1 st and 2nd hours ESR and liver function are significantly higher among cases (Table 5).

SAT titers of cases are $\geq 1320 /$ Vs $\leq 1160 /$ of controls. The differences are significant $(P<0.05$ for each titer except $\geq 12560 /$ ). Meanwhile, the differences between SAT titers of B. abortus and melitensis are insignificant (Table 6).
Cut-off point of SAT titer 1320/ discriminates between cases and controls. Cases lie significantly in AUC with high sensitivity $(96.4 \%)$ and specificity $(100.0 \%)$ (Table 7$)$.

Positive Brucella cultures represent $58.2 \%$ of the cases. There are insignificant differences between SAT titers and blood culture positivity among the cases. Positive and negative IgM results are $69.4 \%$ and $30.6 \%$ of the cases, respectively with statistically insignificant differences except for titer 1640/ $(P=0.03)$. Positive and $-\mathrm{ve} \operatorname{IgG}$ results are $65.3 \%$ and $34.7 \%$ of cases, respectively with statistically insignificant differences at all titers. There is insignificant difference in SAT titers as a whole neither between $\operatorname{IgM}+$ ve and -ve groups nor between $\mathrm{IgG}+\mathrm{ve}$ and $-\mathrm{ve}$ groups. This indicates there isn't association between SAT titers [as one entity] and ELISA results (Table 8).

Table 3: Distribution of the studied cases of brucellosis and controls according to their Occupational-risk factors

\begin{tabular}{|c|c|c|c|c|c|}
\hline \multirow{2}{*}{ Variables } & \multicolumn{2}{|c|}{ Cases $(n=98)$} & \multicolumn{2}{|c|}{ Controls $(n=98)$} & \multirow{2}{*}{$\begin{array}{l}\mathrm{OR}^{*}(95 \% \mathrm{CI})^{* * *} \\
\mathrm{OR}(95 \% \mathrm{ECL})^{* * * *}\end{array}$} \\
\hline & No. & $\%$ & No. & $\%$ & \\
\hline \multicolumn{6}{|l|}{ Occupational exposure: } \\
\hline Contact with animals: & 41 & 41.8 & 13 & 13.3 & $4.7(2.2-10.19)^{*}$ \\
\hline Butcher and slaughtering workers & 14 & 14.3 & 2 & 2.0 & $8.0(1.74-73.91)^{* * *}$ \\
\hline Farmers and dairy workers & 13 & 13.3 & 5 & 5.1 & $3.59(1.05-15.62)^{* * *}$ \\
\hline Veterinarians & 4 & 4.1 & 1 & 1.0 & $4.13(0.4-205.33)^{* * *}$ \\
\hline Meat transporters and driver & 10 & 10.2 & 5 & 5.1 & $2.11(0.63-8.17)^{* * *}$ \\
\hline No contact with animals: & 57 & 58.2 & 85 & 86.7 & $0.21(0.1-0.45)^{*}$ \\
\hline House wife & 30 & 30.6 & 37 & 37.8 & $0.73(0.38-1.37)^{*}$ \\
\hline Student & 12 & 12.2 & 19 & 19.4 & $0.58(0.25-1.35)^{*}$ \\
\hline Clerical work & 9 & 9.2 & 19 & 19.4 & $0.42(0.16-1.05)^{*}$ \\
\hline Others e.g. manual and skilled worker & 6 & 6.1 & 10 & 10.2 & $0.57(0.16-1.83)^{* * *}$ \\
\hline \multicolumn{6}{|l|}{ Duration of occupational exposure (years): } \\
\hline$<5$ & 10 & 10.2 & 7 & 7.1 & $1.48(0.49-4.53)^{*}$ \\
\hline $5-9$ & 11 & 11.2 & 4 & 4.1 & $2.97(0.84-13.21)^{* *}$ \\
\hline $10-19$ & 13 & 13.3 & 3 & 3.1 & $4.84(1.26-27.19)^{* *}$ \\
\hline$\geq 20$ & 24 & 24.5 & 2 & 2.0 & $15.57(3.63-138.58)^{* *}$ \\
\hline
\end{tabular}

*OR: Odds ratio, $\quad * * \mathrm{CI}$ : Confidence interval, $\quad * * * \mathrm{CI}$ : Exact confidence limits 
Table 4: Distribution of the studied cases of brucellosis and controls according to their symptoms and signs

\begin{tabular}{|c|c|c|c|c|c|c|}
\hline \multirow{3}{*}{ Variables } & \multicolumn{4}{|c|}{ The studied groups } & \multirow{3}{*}{ Yates $\chi^{2}$} & \multirow{3}{*}{ P-value } \\
\hline & \multicolumn{2}{|c|}{ Cases $(n=98)$} & \multicolumn{2}{|c|}{ Controls $(\mathrm{n}=98)$} & & \\
\hline & No. & $\%$ & No. & $\%$ & & \\
\hline \multicolumn{7}{|c|}{ Clinical symptoms } \\
\hline Fever, rigor, and/or sweating & 89 & 90.8 & 9 & 9.2 & 127.37 & 0.0000 \\
\hline Muscloskeletal: Joint affection, body-aches, and/or back-pains & 86 & 87.8 & 21 & 21.4 & 84.3 & 0.0000 \\
\hline Headache & 85 & 86.7 & 39 & 39.8 & ${ }^{4} 4.46$ & 0.0000 \\
\hline Anorexia, nausea and/or vomiting & 69 & 70.4 & 21 & 21.4 & 45.38 & 0.00000 \\
\hline Abdominal pains and/or constipation & 58 & 59.2 & 13 & 13.3 & 42.76 & 0.0000 \\
\hline Cough/dyspnea/chest pain & 46 & 46.9 & 12 & 12.2 & 26.67 & 0.0000 \\
\hline Genitourinary symptoms & 38 & 38.8 & 11 & 11.2 & 18.39 & 0.00001 \\
\hline \multicolumn{7}{|c|}{ Clinical signs } \\
\hline High temperature & 87 & 88.8 & 4 & 4.1 & 137.93 & 0.000 \\
\hline Lymph node enlargement (peripheral) & 25 & 25.5 & 9 & 9.2 & 8.01 & 0.004 \\
\hline Pallor & 31 & 31.6 & 13 & 13.3 & 8.47 & 0.003 \\
\hline Jaundice & 14 & 14.3 & 6 & 6.1 & 2.73 & 0.098 \\
\hline \multicolumn{7}{|l|}{ Abdomen: } \\
\hline Hepatomegaly and/or tender liver & 42 & 42.4 & 9 & 9.2 & 27.14 & 0.0000 \\
\hline Splenomegaly and/or tender spleen & 34 & 34.7 & 12 & 12.2 & 12.53 & 0.0004 \\
\hline Chest affection & 32 & 32.7 & 12 & 12.2 & 10.58 & 0.001 \\
\hline Hypertension and/or heart diseases & 24 & 24.5 & 19 & 19.4 & 0.48 & 0.489 \\
\hline Tender and/or swollen joints & 11 & 11.2 & 5 & 5.1 & 1.7 & 0.19 \\
\hline Tender spine & 9 & 9.2 & 4 & 4.1 & 1.32 & 0.25 \\
\hline Swollen and/or tender testes & 21 & 21.4 & 6 & 6.1 & 8.42 & 0.003 \\
\hline
\end{tabular}

Table 5: Distribution of the studied cases of brucellosis and controls according to the results of routine lab tests

\begin{tabular}{|c|c|c|c|c|}
\hline \multirow{3}{*}{ Variables } & \multicolumn{2}{|c|}{ The studied groups } & \multirow{3}{*}{ t-value } & \multirow{3}{*}{ P-value } \\
\hline & Cases $(\mathrm{n}=98)$ & Controls $(\mathrm{n}=98)$ & & \\
\hline & Mean \pm SD & Mean \pm SD & & \\
\hline \multicolumn{5}{|c|}{ Routine lab tests (Mean \pm SD) } \\
\hline \multicolumn{5}{|l|}{ CBC (Mean \pm SD): } \\
\hline $\mathrm{Hb}(\mathrm{mg} / \mathrm{dl})$ & $12.9 \pm 1.6$ & $13.6 \pm 1.7$ & -2.968 & 0.001 \\
\hline RBC (millions/cmm) & $5.2 \pm 0.9$ & $5.6 \pm 0.8$ & -3.288 & 0.0005 \\
\hline WBC (thousands/cmm) & $5.9 \pm 2.7$ & $5.7 \pm 2.6$ & -0.528 & 0.298 \\
\hline \multicolumn{5}{|l|}{ ESR $($ Mean \pm SD): } \\
\hline $1^{\text {st }}$ hour & $27.2 \pm 14.3$ & $21.4 \pm 11.6$ & -3.118 & 0.001 \\
\hline $2^{\text {nd }}$ hour & $41.2 \pm 17.8$ & $34.3 \pm 16.7$ & -2.799 & 0.002 \\
\hline \multicolumn{5}{|c|}{ Liver function tests $($ Mean \pm SD) } \\
\hline T. Serum bilirubin $(\mathrm{mg} / \mathrm{dl}$, Mean $\pm \mathrm{SD})$ & $1.2 \pm 0.1$ & $1.0 \pm 0.1$ & -14.0 & 0.000000 \\
\hline $\operatorname{ALT}(\mathrm{U} / \mathrm{L}, \mathrm{Mean} \pm \mathrm{SD})$ & $50.1 \pm 15.4$ & $32.5 \pm 9.2$ & -9.713 & 0.00000 \\
\hline $\operatorname{AST}(\mathrm{U} / \mathrm{L}, \mathrm{Mean} \pm \mathrm{SD})$ & $51.2 \pm 13.1$ & $35.6 \pm 8.3$ & -9.958 & 0.00000 \\
\hline $\operatorname{ALP}(\mathrm{U} / \mathrm{L}, \mathrm{Mean} \pm \mathrm{SD})$ & $109.6 \pm 35.3$ & $76.9 \pm 21.4$ & -7.842 & 0.00000 \\
\hline \multicolumn{5}{|c|}{ Kidney function tests $($ Mean \pm SD) } \\
\hline Urea (mg/dl, Mean \pm SD) & $30.4 \pm 8.3$ & $28.6 \pm 8.2$ & -1.527 & 0.06 \\
\hline Creatinine $(\mathrm{mg} / \mathrm{dL}, \mathrm{Mean} \pm \mathrm{SD})$ & $0.9 \pm 0.8$ & $0.8 \pm 0.1$ & -1.228 & 0.111 \\
\hline
\end{tabular}

CBC: Complete blood count, Hb: Hemoglobin, RBC: Red blood corpuscle ESR: Erythrocyte sedimentation rate, WBC: White blood cells ALT: Alanine amino-transferase, AST: Aspartate amino-transferase ALP: Alkaline Phosphatase 
Table 6: Distribution of the studied cases of brucellosis and controls according to results of standard agglutination test (SAT) titer

\begin{tabular}{|c|c|c|c|c|c|c|}
\hline \multirow{3}{*}{$\mathrm{SAT}^{*}$ titer } & \multicolumn{4}{|c|}{ The studied groups } & \multirow{3}{*}{$\chi^{2} \mathrm{FE}^{* *}$} & \multirow{3}{*}{ P-Value } \\
\hline & \multicolumn{2}{|c|}{ Cases $(n=98)$} & \multicolumn{2}{|c|}{ Controls $(\mathrm{n}=98)$} & & \\
\hline & No. & $\%$ & No. & $\%$ & & \\
\hline$\leq 1 / 80$ & 0 & 0.0 & 86 & 87.8 & 149.69 & 0.000 \\
\hline $1 / 160$ & 0 & 0.0 & 12 & 12.2 & 10.74 & 0.001 \\
\hline $1 / 320$ & 12 & 12.2 & 0 & 0.0 & 10.74 & 0.001 \\
\hline $1 / 640$ & 36 & 36.7 & 0 & 0.0 & 41.68 & 0.000 \\
\hline $1 / 1280$ & 46 & 46.9 & 0 & 0.0 & 57.52 & 0.000 \\
\hline \multirow[t]{2}{*}{$\geq 1 / 2560$} & 4 & 4.1 & 0 & 0.0 & $\mathrm{FE}$ & 0.121 \\
\hline & \multicolumn{4}{|c|}{ Discovered brucella species $\left(\mathrm{n}=140^{* * *}\right)$} & & \\
\hline \multirow[t]{2}{*}{ SAT titer } & \multicolumn{2}{|c|}{ Abortus $(\mathrm{n}=80=81.6 \%)$} & \multicolumn{2}{|c|}{ Melitensis $(\mathrm{n}=60=61.2 \%)$} & $x^{2}$ & P-value \\
\hline & No. & $\%$ & No. & $\%$ & & \\
\hline $1 / 320$ & 11 & 13.8 & 6 & 10.0 & 0.17 & 0.681 \\
\hline $1 / 640$ & 29 & 36.2 & 24 & 40.0 & 0.08 & 0.782 \\
\hline $1 / 1280$ & 36 & 45.0 & 28 & 46.7 & 0.0 & 0.98 \\
\hline$\geq 1 / 2560$ & 4 & 5.0 & 2 & 3.3 & $\mathrm{FE}$ & 0.7 \\
\hline
\end{tabular}

*SAT: Standard agglutination test, $\quad$ **FE: Fisher exact test,$\quad * * * 42$ mixed infection cases, B. abortus and melitensis

Table 7: SAT predictive ability to discriminate Brucella cases using receiver operated characteristic (ROC) curve

\begin{tabular}{cccccc}
\hline \multicolumn{5}{c}{ SAT predictive ability to discriminate Brucella cases from controls } \\
\hline AUC* & $95 \% \mathrm{CI}^{* *}$ & P-value & Titer cut-off point & Sensitivity & Specificity \\
0.98 & $0.96-0.99$ & 0.0001 & $1 / 320$ & $96.4 \%$ & $100.0 \%$ \\
\hline
\end{tabular}

*AUC: Area under the ROC curve

**CI: Confidence interval

Table 8: Distribution of the results of blood cultures, and immunoglobulin (Ig) M and G among the studied cases of brucellosis according to standard agglutination test (SAT)

\begin{tabular}{|c|c|c|c|c|c|c|}
\hline \multirow{3}{*}{$\mathrm{SAT}^{*}$ titer } & \multicolumn{4}{|c|}{ Blood culture $(\mathrm{N}=98)$} & \multirow{3}{*}{$\chi 2 \mathrm{FE}^{* *}$} & \multirow{3}{*}{ P-Value } \\
\hline & \multicolumn{2}{|c|}{ Positive $(\mathrm{N}=57=58.2 \%)$} & \multicolumn{2}{|c|}{ Negative $(\mathrm{N}=41=41.8 \%)$} & & \\
\hline & No. & $\%$ & No. & $\%$ & & \\
\hline $1 / 320$ & 4 & 7.0 & 5 & 12.2 & $\mathrm{FE}$ & 0.484 \\
\hline $1 / 640$ & 22 & 38.6 & 10 & 24.4 & 1.59 & 0.207 \\
\hline $1 / 1280$ & 26 & 45.6 & 25 & 61.0 & 1.68 & 0.194 \\
\hline \multirow[t]{2}{*}{$\geq 1 / 5120$} & 5 & 8.8 & 1 & 2.4 & FE & 0.395 \\
\hline & \multicolumn{4}{|c|}{ IgM results $(\mathrm{n}=98)$} & \multirow{3}{*}{$\chi^{2}$} & \multirow{3}{*}{ P-value } \\
\hline \multirow[t]{2}{*}{ SAT titer } & \multicolumn{2}{|c|}{ Positive $(n=68=69.4 \%)$} & \multicolumn{2}{|c|}{ Negative $(n=30=30.6 \%)$} & & \\
\hline & No. & $\%$ & No. & $\%$ & & \\
\hline $1 / 320$ & 12 & 17.6 & 5 & 16.7 & 0.03 & 0.863 \\
\hline $1 / 640$ & 28 & 41.2 & 5 & 16.7 & 4.56 & 0.032 \\
\hline $1 / 1280$ & 22 & 32.4 & 14 & 46.7 & 1.27 & 0.259 \\
\hline$\geq 1 / 2560$ & 3 & 4.4 & 2 & 13.3 & FE & 0.195 \\
\hline \multirow[t]{2}{*}{$\geq 1 / 5120$} & 3 & 4.4 & 1 & 6.6 & FE & 0.64 \\
\hline & \multicolumn{3}{|c|}{$\chi 2=7.41$} & alue $=0.1$ & & \\
\hline \multirow{3}{*}{ SAT titer } & \multicolumn{4}{|c|}{$\operatorname{IgG}(\mathrm{N}=98)$} & \multirow{3}{*}{$\chi^{2}$} & \multirow{3}{*}{$P$-value } \\
\hline & \multicolumn{2}{|c|}{ Positive $(\mathrm{N}=64=65.3 \%)$} & \multicolumn{2}{|c|}{ Negative $(\mathrm{N}=34=34.7 \%)$} & & \\
\hline & No. & $\%$ & No. & $\%$ & & \\
\hline $1 / 320$ & 10 & 15.6 & 7 & 20.6 & 0.11 & 0.735 \\
\hline $1 / 640$ & 23 & 35.9 & 10 & 29.4 & 0.18 & 0.67 \\
\hline $1 / 1280$ & 21 & 32.8 & 13 & 38.2 & 0.1 & 0.753 \\
\hline$\geq 1 / 2560$ & 6 & 9.4 & 3 & 8.8 & FE & 1.0 \\
\hline \multirow[t]{2}{*}{$\geq 1 / 5120$} & 4 & 6.3 & 1 & 2.9 & $\mathrm{FE}$ & 0.655 \\
\hline & \multicolumn{3}{|c|}{ *SAT: Standard agglutination test, } & \multicolumn{2}{|c|}{ **FE: Fisher exact test } & \\
\hline
\end{tabular}




\section{DISCUSSION}

This study showed $65.3 \%$ of suspected patients proved, using SAT, to have brucellosis, which is the commonest infection causes $\mathrm{FUO}^{[12]}$. Prakash et al. ${ }^{[24]}$ found $25.7 \%$ seropositivity of Brucella Abs in FUO patients. Our figure is much higher; our patients were clinically and epidemiologically potential cases. Basyony et al. ${ }^{[25]}$ cleared $82.3 \%$ of patients were seropositive. We found B. abortus the commonest pathogen, 38.8\%; Pappas et al. ${ }^{[16]}$ cleared majority of the cases worldwide were B. melitensis. Our result might be explained, animal hosts of B. abortus are cows\& buffalos that common in Egypt. Also, Abdelbaset et al. ${ }^{[26]}$ found $80.0 \%$ of the positive reactors had B. Abortus only and $20.0 \%$ had mixed infection. While, El-Hamshary et al. ${ }^{[27]}$ reported infection with B. melitensis and Abortus were 49.4\% and 30.4\%, respectively in Banha Fever Hospital. Further, Elbeltagy ${ }^{[28]}$ showed $13.9 \%, 44.5 \%$, and $40.9 \%$ of their patients had B. abortus, melitensis, and mixed, respectively. Most (38.8\%) of our cases were presented in the summer months. This result is consistent with Fouad et al. ${ }^{[29]}$ and Abd-Elall ${ }^{[30]}$. We reported mean total time of antibiotics' use was 26.7457.68 \pm day; Yang ${ }^{[2]}$ cleared sufficient period of drug therapy, 6weeks-6months, has significant role in cure achievement. Our short antimicrobial time use could be explained; high cost and socio-cultural factors. We showed relapse occurred in $26.5 \%$ of cases. Gotuzzo $^{[4]}$ cleared after antimicrobial therapy, $10.0 \%$ of the patients experienced relapse. Our high relapse rate could be explained; therapy discontinuation (short period and/or intermittent use) and continuous exposure to infection in high risk groups. Further, in tuberculosis-endemic populations as Egypt, community-acquired rifampin resistance should be taken into account in brucellosis treatment.

We cleared the older age was significant riskfactor. Our result agrees with Al-Sekait ${ }^{[31]}$, he showed age $\geq 45$ year was significant risk associated with seropositivity. Hussein et al. ${ }^{[32]}$ found brucellosis increased among patients aged 41 - 50 year. Further, Tumwine et al. ${ }^{[6]}$ cleared $22.2 \%$ of patients were $>60$ year. On contrary, Al-Tawfiq and Abukhamsin ${ }^{[33]}$ found patients aged 2040-years had the highest rate. Also, Fallatah et al. ${ }^{[34]}$ observed $60.3 \%$ of the patients were 1340-year. Meanwhile, $14.3 \%$ of our patients aged up-to 18 year. Gotuzzo ${ }^{[4]}$ cleared brucellosis in school-aged children, worldwide, accounts for up-to $10.0 \%$. But, it's up-to $20.0 \%-25.0 \%$ in endemic areas. However, Abdelbaset et al. ${ }^{[26]}$ found insignificant risk of age on contracting brucellosis; individuals in age group 35-63years had increased risk of exposure compared to younger age group.

We found male gender was significant riskfactor. Worldwide, males have higher prevalence of brucellosis, that is constant epidemiological feature $^{[6,26,33,34,35]}$. Our result agrees with this feature, which could be explained; types of males' occupations and the differences in the practice and habits. Fouad et $a l .{ }^{[29]}$ found $70.0 \%$ of the patients were males. On contrary, Hussein et al. ${ }^{[32]}$ showed brucellosis prevalence was significantly higher in females. Also, Abdelbaset et al. ${ }^{[26]}$ found insignificant risk of the male gender in acquiring brucellosis.

We showed rural residence was significant riskfactor $(\mathrm{OR}=2.86)$. Our result agrees with Al-Sekait ${ }^{[31]}$ and Tumwine et al. ${ }^{[6]}$; they reported significant riskfactors $(\mathrm{OR}=2.8$ and 3.16, respectively). On contrary, Fouad et al. ${ }^{[29]}$ observed $75.5 \%$ of their patients were urban residents $(p<0.01)$. Minas et al. ${ }^{[35]}$ showed urban population isn't at great risk to acquire brucellosis; commercial dairy-products were manufactured from pasteurized milk.

We noticed illiteracy, unskilled labor, and low socioeconomic level were significant risk-factors. The most affected population are the poorly educated ${ }^{[18]}$. Al-Sekait ${ }^{[31]}$ found unskilled labor was significant risk-factor $(\mathrm{OR}=3.8)$. While, Elbeltagy ${ }^{[28]}$ cleared $54.0 \%$ and $44.5 \%$ of patients had no- and moderateeducation, respectively. Tumwine et al. ${ }^{[6]}$ showed most of the cases had no- or primary-education. On contrary, Abdelbaset et al. ${ }^{[26]}$ showed illiterates were insignificant risk-factor to catch brucellosis. Cetinkaya et al. ${ }^{\left[{ }^{[6]}\right.}$ found brucellosis wasn't related to educational level. These results lightened the need for healtheducation program for such risky group.

Regarding lifestyle risks; eating cottage-cheese, drinking raw- and/or un-pasteurized milk, eating polluted ice-cream, breeding animals at home, and slaughtering animals were significant risk-factors. The organisms may survive in un-pasteurized goat cheese for up-to 8weeks. Freezing dairy-products or meat doesn't destroys the organisms that are killed by pasteurization and boiling ${ }^{[37]}$. Consumption of rawmilk and milk-products were the most prevalent riskfactors $^{[29]}$; Al-Sekait ${ }^{[31]}$ reported 5.5 significant risk for drinking raw-milk. Further, Saleh ${ }^{[38]}$ cleared $54.1 \%$ of patients had history of raw-milk ingestion. Tumwine et al. $^{[6]}$ elicited consuming milk-products and locally processed milk-products were significant risk-factors ( $\mathrm{OR}=2.36,2.54$; respectively). On contrary, Minas et al. ${ }^{[35]}$ showed $8.5 \%$ of their cases infection was attributed to consumption of dairy-products. Also, Meky et $a l{ }^{[39]}$ found drinking raw-milk and eating cottage-cheese were insignificant risk-factors, while eating polluted ice-cream and breeding animals at home were significant risk-factors $(\mathrm{OR}=1.8,2.3$; respectively). On contrary, Tumwine et al. ${ }^{[6]}$ showed breeding animals at home was insignificant riskfactor. We found family history of similar attack was significant risk-factor. Household members of patients may have been exposed to the pathogen and became infected/ill[ ${ }^{[40]}$. More than $1 / 3(37.6 \%)$ of the patients 
had positive family history[37].

Brucella may transmitted to man through direct contact with infected animals or their secretions ${ }^{[14]}$. Brucellosis is usually related to occupational exposure ${ }^{[4]}$; some occupations were proved to be riskfactors as ranchers, veterinarians, and abattoir- and lab-workers ${ }^{[14]}$. Brucellosis is considered an important occupational disease ${ }^{[41]}$. We showed occupations with animal contact were significant risk-factors for acquiring brucellosis. Our result is compatible with Elbeltagy ${ }^{[28]}$, Fouad et al. ${ }^{[29]}$, Abd-Elall ${ }^{[30]}$, Minas et $a l .{ }^{[35]}$, Saleh ${ }^{[38]}$, Meky et al. ${ }^{[39]}$, Farghaly et al. ${ }^{[41]}$; they elicited close contact with animals or their products was the commonest feature/significant risk-factor. Mishal et $a l .{ }^{[42]}$ showed almost all of the infected patients worked in cowshed, participated in calf deliveries, and had contact with cows' bloods and placentas. Meky et $a l .{ }^{[39]}$ and Fouad et al. ${ }^{[29]}$ cleared farmers, butchers, and meat-transporter workers\& vehicle-drivers were commonest occupations at risk. Saleh ${ }^{[38]}$ reported direct contact with animals was found in $45.6 \%$ of the patients. Further, Prakash et al. ${ }^{[24]}$ showed Brucella seropositivity was $37.1 \%$ in milkman and $26.7 \%$ in meat handlers/veterinarians. On contrary, Tumwine et $a l^{[6]}$ elicited contact with animals and slaughter animals were insignificant risk-factors. Further, we noticed occupations with no animal contact were significant protective factor. This result is expected and accepted as occupations that not exposing the subject to risk of infection might be decrease probability of infection. Also, we found the longer duration of occupational exposure the higher significant risk of disease. Again, this result is expected and accepted; there was a tendency towards increase infection rate with increase duration of exposure. Mahgoub ${ }^{[43]}$ found $38.9 \%$ of seropositive workers exposed to risk of brucella infection for $\geq 5$ years. While, Refaat et al. ${ }^{[44]}$ didn't find significant difference between veterinarians working more or less than 10 years.

We viewed most of the patients had many nonspecific symptoms and signs. Brucellosis is a multisystem disease that can manifest with a broad spectrum of clinical features as fever, headache, back-pain, weakness, profuse sweating, chills, and joint-pain, etc. Fever is common symptom and sign; $72.0 \%-91.0 \%{ }^{[2,45]}$. The most observed symptoms were fever $(94.6 \%)$, fatigue $(92.8 \%)$, body-ache $(91.4 \%)$, sweating $(87.4 \%)$, joint-pain $(86.2 \%)$, back-pain $(86.2 \%)$, chills $(82.0 \%)$, headache $(80.6 \%)$, loss of appetite $(77.6 \%)$, weight-loss $(65.2 \%)$, constipation $(64.9 \%)$, abdominal-pain $(45.0 \%)$, sleep-disturbances $(37.0 \%)$, and cough $(24.4 \%)^{[35]}$. While, the commonest signs were tender-spine $(48.0 \%)$, arthritis $(40.4 \%)$, lymphadenopathy $(32.0 \%)$, splenomegaly $(25.0 \%)$, pallor $(22.0 \%)$, and epididymoorchitis $(21.3 \%)^{[37]}$. Also, Fouad et al. ${ }^{[29]}$ viewed the commonest symptoms and signs were fever $(98.7 \%)$, weakness
$(80 \%)$, profuse sweating $(74.7 \%)$, abdominal-pain $(72 \%)$, and $(34.9 \%)$. Further, Ruiz-Mesa et al. ${ }^{[46]}$ found hepatomegaly and splenomegaly were $35.2 \%$ and $20.8 \%$, respectively. Furthermore, El-Moselhy et $a l .{ }^{[45]}$ viewed most of symptoms were significantly more frequent among the patients than controls.

We showed mean $\mathrm{Hb}$ level and RBCs count were significantly lower among cases than controls, while mean ESR at $1^{\text {st }} \& 2^{\text {nd }}$ hour, and liver functions were significantly higher among cases than controls. Meanwhile, means of WBCs count and kidney functions were insignificantly higher among cases. Young ${ }^{[14]}$ cleared routine laboratory tests aren't particularly helpful. Anemia and leucopenia are common findings. The WBCs count is often normal or low and may not suggest an infectious process. The ESR is variable and of little diagnostic value. Our results regarding mild liver functions impairment during the course of brucellosis are agreed with LaSpada et al. ${ }^{[47]} ; 38.0 \%$ and $53.0 \%$ of patients had elevated baseline values of AST and ALT, respectively.

SAT could be considered a confirmatory test for other screening laboratory tests ${ }^{[48]}$. Brucellosis should be considered in individuals with unexplained chronic fever and non-specific complaints ${ }^{[2]}$. SAT titer $\geq 1160 /$ is considered diagnostic as long as the patient has signs and symptoms of disease. However, in endemic areas the diagnostic threshold value has to be 1320 / to provide sufficient high specificity ${ }^{[4]}$. We reported SAT titer $\geq 1320$ / in all cases. Meanwhile, the entire control group SAT titer was $\leq 1160 /$. The cut-off point of SAT titer between cases and controls in current study was $1320 /$. This indicates that +ve SAT at titer $\leq 1160$ / is common in healthy subjects because Brucella is endemic in Egypt leading to repeated exposure of the populations, particularly high risk groups, to infection. In endemic areas, titer $\geq 1320 /$ is recommended in the diagnosis of brucellosis ${ }^{[2]}$. Further, we noticed SAT titer 1320/ among the patients lies significantly in the AUC ROC with high sensitivity (96.4\%) and specificity $(100.0 \%)$. These results are similar to AbdElall ${ }^{[30]}$ and Zaky et al..$^{[50]}$. Also, Cakan, et al. ${ }^{[51]}$ showed sensitivity and specificity of SAT is $95.6 \%$ and $100 \%$, respectively, which are similar to our results.

We found $58.2 \%$ of blood cultures were positive. The sensitivity of blood culture varies depending on the quantity of bacteria in blood, specimen type, and the used methods; it varies from $15.0 \%-70.0 \%{ }^{[2,52]}$ up-to $90.0 \%{ }^{[4]}$. The difference between our figure and these figures might be because our patients received many antibiotics therapy before diagnosis was confirmed. There were no statistically significant differences between SAT and blood culture positivity among the patients. Absolute diagnosis of brucellosis requires isolation of the bacterium from blood ${ }^{[14]}$. Also, Kiel\& Khan $^{[53]}$ clarified although the cultures are not always positive; blood cultures have $50.0 \%-80.0 \%$ 
sensitivity. So, diagnosis depends on serology, since cultures are not always positive. While, Ruiz-Mesa et al. ${ }^{[46]}$ observed blood cultures were positive in $62.6 \%$ of the patients, while $37.4 \%$ of them were diagnosed according to clinical and serological criteria. On contrary, our result was higher than Abd-Elall[30]; he reported $37.0 \%$ positive cultures.

We observed Brucella +ve IgM and IgG results were found among $69.4 \%$ and $65.3 \%$ of our cases. As IgM Abs appear earlier than IgG Abs, the detection of $\operatorname{IgM}$ in serum is the widely used approach for early serologic diagnosis of acute infection ${ }^{[14]}$; specific IgM Abs dominates during the acute phase of disease. ELISA discriminates between presence of specific $\operatorname{IgM}$ and $\operatorname{IgG} \mathrm{Abs}$ and accesses illness stage ${ }^{[49]}$. ELISA has proved useful; many studies used it as confirmatory test for Brucella screening tests as Rose-Bengal Plate test ${ }^{[54]}$. Abd-Elall[ ${ }^{[30]}$ found ELISA IgM and IgG were positive in $63.0 \%$ and $64.2 \%$ of cases, respectively. Also, Aranís et al. ${ }^{[55]}$ cleared $80.0 \%$ and $50.0 \%$ of patients were ELISA IgG and IgM positive, respectively. Brucella ELISA test is considered to have higher sensitivity and specificity in determining Brucella specific Abs than other serological tests ${ }^{[56]}$. Also, ELISA had higher specificity and sensitivity compared with $\mathrm{SAT}^{[2]}$. However, Cakan et al. ${ }^{[51]}$ showed ELISA test for brucellosis is more sensitive only when both $\operatorname{IgG}$ and IgM were used, though their titer alone didn't represent disease status. Awah-Ndukum et al. ${ }^{[57]}$, Sanogo et al. ${ }^{[58],}$ and Gatechew et al. ${ }^{[59]}$ reported sensitivity and specificity of ELISA IgG were $95.6 \% \& 97.1 \%, 96.1 \% \& 95 \%$, and $96.8 \% \& 96.3 \%$; respectively.

\section{CONCLUSIONS AND RECOMMENDATIONS}

Brucellosis has many important sociodemographic, lifestyle, and clinical risk-factors. Diagnosis of brucellosis depends on presence of risk-factors, clinically suspected, and SAT titer $\geq 1320$ /. Titer $\geq 1320$ / has high sensitivity and specificity. There are no significant relations neither between SAT titer and blood culture results nor IgM and IgG results. More studies are needed to define brucellosis seroprevalence in different areas and situations in Egypt and to understand the full epidemiology of this public health problem.

\section{ACKNOWLEDGEMENT}

We would like to acknowledge the staff members of Assiut Fever Hospital for their generous help in completing this research. Also, we thank the participants in this research for their cooperation.

\section{CONFLICTS OF INTEREST}

There are Conflicts of Interest

\section{REFERENCES}

1. Seleem MN, Boyle SM and Sriranganathan N (2010) Brucellosis: a re-emerging zoonosis. Veterin Microbiol 140 (3 / 4):392-8.
2. Yang X (2016) Infectious diseases- Brucellosis. In: Bope ET and Kellerman RD (ed), Conn's Current Therapy, Section 3, Elsevier Inc, Philadelphia, US, ISBN: 978-0-323-35535-3, PP. 81-83.

3. Bosilkovski M, Dimzova M and Grozdanovski K (2009) Natural history of brucellosis in an endemic region in different time periods. Acta Clin Croat 48:41-6.

4. Gotuzzo E (2012) Brucellosis. In: Goldman L and Schafer A, Goldman's Cecil Medicine, 24th Ed, Section 318, Saunders, Philadelphia, ISBN: 978-1-4377-1604-7.

5. Pappas G, Panagopoulou P, Christou L and Akritidis N (2006) Brucella as a biological weapon. Cell Mol Life Sci 63:22-9.

6. Tumwine G, Matovu E, Kabasa JD, Owiny DO and Majalija S (2015) Human brucellosis: sero-prevalence and associated risk factors in agro-pastoral communities of Kiboga District, Central Uganda. BMC Public Health 15:900-7.

7. Pappas G, Papadimitrious $\mathrm{P}$, Kritidis N, Christou L and Tsian E (2006) The new global map of human brucellosis. Lancet Infect Dis, 6(2): 91-9. DOI: 10.1016/S1473.

8. Southwick F (2014) Reemerging bacterial infections, Brucellosis. Infectious diseasesA clinical short course, 3rd Ed, Chapter 13, McGraw-Hill Medical, ISBN: 978-0-07178925-7, PP: 356-8.

9. Eltholth MM, Hegazy YM, El-Tras WF, Bruce M, Rushton J (2017) Temporal analysis and costs of ruminant brucellosis control program in Egypt between 1999 and 2011. Transbound Emerg Dis 64:1191-9.

10. El-Sherbini A, Kabbash I, Schelling E, Shennawy SE, Shalapy N (2007) Seroprevalences and local variation of human and livestock brucellosis in two villages in Gharbia governorate, Egypt. Trans R Soc Trop Med Hyg 101:923-8.

11. El-Diasty MM, Ahmed HA, Sayour AE, El HofyI, Tahoun AB, Shafik SM (2016) Seroprevalence of Brucella spp. in cattle, molecular characterization in milk, and the analysis of associated risk factors with seroprevalence in humans, Egypt. Vector Borne Zoonotic Dis 16(12):758-64.

12. Ali-Eldin FA, Abdelhakam SM and AliEldin ZA (2011) Clinical spectrum of fever of unknown origin among adult Egyptian patients admitted to Ain-Shams University Hospitals: a hospital based study. J Egypt Soc Parasitol 41:379-86. 
13. Afifi S, Earhart K, Azab M, Youssef F, ElSakka H, Wasfy M, et al (2005) Hospital-based surveillance for acute febrile illness in Egypt: a focus on community-acquired bloodstream infections. Am J Trop Med Hyg 73:392-9.

14. Carroll KC and Hobden JA (2016) Brucella. In: Carroll KC, Hobden JA, Miller S, Morse SA, Mietzner TA, Detrick, et al (eds), Jawetz Melnick \& Adelberg's medical microbiology, $27^{\text {th }}$ Ed, Mcgraw-Hill Education: New York.

15. Colmenero JD, Reguera JM, Martos F, Sánchez-De-Mora D, Delgado M, Causse M, et al (1996) Complications associated with Brucella melitensis infection: a study of 530 cases. Medicine, 75:195-211.

16. Pappas G, Akritidis N, Bosilkovski $M$ and and Tsianos E (2005) Brucellosis. NEJM, 352:2325-36

17. Innes AJ (2016) Infectious diseases. Davidson's essentials of medicine, 2nd Ed, Chapter 5, Churchill Livingstone- Elsevier, ISBN: 978-07020-5592-8, PP. 47-52.

18. Ayoola MC, Akinseye VO, Cadmus E, Awosanya E, Popoola OA, Akinyemi OO, et al (2017) Prevalence of bovine brucellosis in slaughtered cattle and barriers to better protection of abattoir workers in Ibadan, South-Western Nigeria. Pan African Medical J 28:68, DOI:10.11604/pamj.2017.28.68.10925.

19. Serra J and Viñas M (2004) Laboratory diagnosis of brucellosis in a rural endemic area in northeastern Spain. Int Microbiol 7(1):53-8.

20. Baddour MM (2012) Diagnosis of brucellosis in humans: a review. J Veterin Advances 2(4):149-56.

21. El-Gilany A, El-Wehady A, El-Wasify $M$ (2012) Updating and validation of the socioeconomic status scale for health research in Egypt. EMHJ 18: 962-8.

22. Salata RA (1996) Review of human brucellosis. J Infect Dis 174:1720-7.

23. Voller A, Bartlett A and Bidwell DE (1978) Enzyme immunoassays with special reference to ELISA techniques. J Clin Pathol 31 (6):507-20.

24. Prakash P, Bhansali S, Gupta E, Kothari D, Mathur A, and Ambuwani S (2012) Epidemiology of brucellosis in high risk group and PUO patients of western-Rajasthan. Nat J Community Med 3(1):61-5.

25. Basyony AF, Aboulwafa MM, Hafez MM and Abou Gazia KA (2012) Comparative study on serodiagnostic techniques of brucella infection in Egypt. Arch Clin Microbiol 3(2/3), DOI: $10.3823 / 251$.

26. Abdelbaset AE, Abushahba MFN, Hamed MI and Rawy MS (2018) Sero-diagnosis of brucellosis in sheep and humans in Assiut and El-Minya governorates, Egypt. International J Veterin Scie Med 6(2018):S63-S67.

27. El-Hamshary NK, Ammar EG, Abd El-Aziz IM, El-Sayed AM, Mohmmed AA and Ahmed MU (2008) Seroepidemiological study of human brucellosis among patients admitted in Banha Fever Hospital. AAMJ 6(1):137-44.

28. Elbeltagy KE (2001) An epidemiological profile of brucellosis in Tabuk province, Saudi Arabia. EMHJ 7(4/5):791-8.

29. Fouad A, El-Akel W, Abdel Hafez H and Abdullah R (2011) Update of brucellosis in Egyptian referral fever hospital. Kasr El Aini Med J 17(2):51-57.

30. Abd-Elall MA (2013) Current status of Brucella infections in a major referral fever hospital in Egypt. MD Thesis in Tropical Medicine, Faculty of Medicine, Al-Azhar University.

31. Al-Sekait MA (1999) Seroepidemiologicl survey of brucellosis antibodies in Saudi Arbia. Ann Saudi Med 19(3):219-22.

32. Hussein AA, Sayed AS and El-Feki MA (2005) Seroepidemiological study on human brucellosis in Assiut Governorate. Egypt J Immun 12(1):49-56.

33. Al-Tawfiq JA and Abukhamsin A (2009) Burden and etiology of community acquired bacterial meningitis in hospital in Eastern Saudi Arabia: 1993-2006. Med Sci Monit 15(2):10-4.

34. Fallatah SM, Oduloju AJ, Al-Dusari SN and Fakunle YM (2005) Human brucellosis in Northern Saudi Arabia. Saudi Med J 10:1562-6.

35. Minas M, Minas A, Gourgulianis $\mathrm{K}$ and Stournara A (2007) Epidemiological and clinical aspects of human brucellosis in Central Greece. Jpn J Infect Dis, 60(6):362-6.

36. Cetinkaya Z, Aktepe OC, Ciftci IH and Demire R (2005) Seroprevalence of human brucellosis in a rural area of Western Anatolia, Turkey. J Health Popul Nutr 23:137-41.

37. Beeching NJ and Madkour MM (2014) Bacterial infection: Brucellosis. In: Farrar J, Hotez P, Junghanss T, Kang G, Lalloo D, White NJ (eds.), Manson's tropical diseases. 23nd Ed, Saunders, Elsevier, ISBN: 978-0702-501-2, PP. 371-8. 
38. Saleh M (2014) Prevalence of Malta fever in Assiut Fever hospital. Master Thesis in Internal Medicine, Faculty of Medicine, Assiut, AlAzhar University.

39. Meky FA, Hassan EA, Abd Elhafez AM, Aboul Fetouh AM and El-Ghazali SM (2007) Epidemiology and risk factors of brucellosis in Alexandria governorate. EMHJ 13(3):677-85.

40. Almuneef MA, Memish ZA, Balkhy $\mathrm{HH}$, Alotaibi B, Algoda S, Abbas M et al (2004) Importance of screening household members of acute brucellosis cases in endemic areas. Epidemiol Infect 132:533-40.

41. Farghaly RM, Amer SA, Fahim AE, Kishk RM and Abbas MF (2018) Predictors of brucellosis seropositivity among exposed workers. Egypt J Occup Med 42(2):209-26.

42. Mishal J, Ben Israel N, Levin Y, Sherf S, Jafari J, Embon E, et al (1999) Brucellosis outbreak: analysis of risk factors and serological screening. Int J Mol 4:655-8.

43. Mahgoub KG (1995) Occupational brucellosis in Egypt. MD Thesis in Industrial Medicine and Occupational Diseases, Faculty of Medicine, Cairo University.

44. Refaat A, Abou-El-Fotoh O, El-Sobky MK, Abdel-Rasoul GM, Farahat MF (1998) Screening for brucellosis in occupationally exposed veterinarians in Menoufiya governorate. Egypt J Occup Med 22(2):185-95.

45. El-Moselhy EA, Zayet H, El-Khateeb AS, Mohammed AS, El-Tiby D, Abd ElAziz A, et al (2018) Human Brucellosis: Seroprevalence, Risk Factors, and Barriers of Protection among Slaughterhouses' Workers in El-Menia Governorate, Egypt. Archive Clin Path 1002.

46. Ruiz-Mesa JD, Sanchez-Gonzalez J, Reguera JM, Martín L, Lopez-Palmero S and Colmenero JD (2005) Rose Bengal test: diagnostic yield and use for the rapid diagnosis of human brucellosis in emergency departments in endemic areas. Clin Microbiol Infect 11: 221-5.

47. LaSpada E, Micalizzi A, La Spada M, Quartarano P, Nugara G, Soresi M, et al (2008) Abnormal liver function in brucellosis. Infec Med 16(3):148-53.

48. Munoz PM, Marin CM, Morreal D, González D, Garin-Bastuji B, Díaz R, et al (2005) Efficacy of several serological tests and antigens for diagnosis of bovine brucellosis in the presence of false-positive serological results due to Yersinia enterocolitica O:9. Clin Diag Lab Immunol 12:141-51.
49. Smits HL and Kadri SM (2005) Brucellosis in India: a deceptive infectious disease. Indian $\mathbf{J}$ Med Res 122:375-84.

50. Zaky S, Azab M, Mansour R, El-Moselhy EA, Bersy A, Omar N, et al (2019) Cutoff point for standard tube agglutination test in the diagnosis of human brucellosis in Egypt. J Advanced Research 16:162-163 [Abstract, DOI:10.1016/j.jare.2019.02.026].

51. Cakan G, Bezirci FB, Kacka A, Cesur S, Aksaray S, Tezeren D, et al (2008) Assessment of diagnostic enzyme-linked immunosorbent assay kit and serological markers in human brucellosis. Japan J Infec Dis 61(5):366-70.

52. Ykan AH (2008) The value of culture and serological methods in the diagnosis of human brucellosis. Mikrobiyol Bul 42(1):185-95.

53. Kiel WF and Khan Y (1987) Analysis of 506 consecutive positive serologic tests for brucellosis in Saudi Arabia. J Clin Microbiol 25(8):1384-7.

54. Salman AMA, Mustafa EA, Hamid AM and Hassan LM (2014) Application of different serological tests for the detection of the prevalence of bovine brucellosis in lactating cows in Khartoum State, Sudan. J Applied Industrial Sciences 2(5):213-8.

55. Aranís JC, Oporto CJ, Espinoza M, Riedel KI, Pérez CC and García CP (2008) Usefulness of the determination of IgG and IGM antibodies by ELISA and immunocapture in a clinical series of human brucellosis. Rev Chilena Infectol 25(2):116-21.

56. Varshochi M, Majidi J, Amini M, Ghabili K and Shoja MM (2011) False positive seroreactivity to brucellosis in tuberculosis patients: A prevalence study. J Gen Intern Med 4:207-10.

57. Awah-Ndukum J, Mouiche MMM, Bayang HN, NguNgwa V, Assana E, Feussom KJM, et al (2018) Seroprevalence and associated risk factors of brucellosis among indigenous cattle in the Adamawa and North Regions of Cameroon. Veterin Med Internatl ID: 3468596.

58. Sanogo M, Thys E and Achietal YL (2013) Bayesian estimation of the true prevalence, sensitivity and specificity of the Rose Bengal and indirect ELISA tests in the diagnosis of bovine brucellosis. Veterin J 195(1):114-20.

59. Getachew T, Getachew G, Sintayehu G, Getenet M, and Fasil A (2016) Bayesian estimation of sensitivity and specificity of Rose Bengal, complement fixation, and indirect ELISA tests for the diagnosis of bovine brucellosis in Ethiopia. Veterin Med internatl ID: 8032753. 


\section{الملخص العربى}

\section{داء البروسيلا البشرى: طرق التشخيص وعوامل الخطوره لاى المرضى المصريين فى مستثفى حميات أسيوط}

عصام عبد المنعم المصيلحى'، تصسين سليمان"، أيمن عبد العزيز عبد الرحمن"،

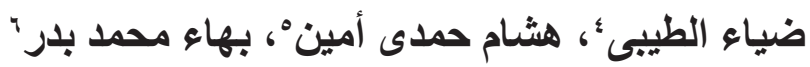

'قسم طب المجتمع، كلية الطب، "ققم الطب الباطني، •قسم الباثولوجيا الإكلينيكية،

"قسم الأحياء الدقيقة الطبية والمناعة، جامعة الأزهر، أسيوط، مصر

'ققم طب المجتمع، ؛قسم الطب الاستثـارى، كلية الطب، جامعة الأزهر، القاهرة، مصر

خلفية: داء البروسيلا البشرى، هو مرض شائع حيو انى المنشأ، ويمثل مشكلة صحية عامة رئيسية في العديد من البلدان في جميع أنحاء العالم بما في ذلك مصر. الأهداف: تحديد عو امل الخطوره لمرضى داء البروسيلا وتقييم الطرق المختبريه لتشخيص مرض البروسيلا في مستثفى حميات أسيوط. المرضى وطرق البحث: جندت الدر اسة 98 مريضا يعانون من داء البروسيلات و عدد متساو من الاشخاص الأصحاء كمجمو عة ضابطه. تم إخضاع جميع المشاركين للمقابلة، والفحص السريري، و الفحوصات المخبرية. النتائج: كان كبار السن، الذكور، و الإقامة الريفية، و الحالة الإجتماعية والإقتصادية المنخفضة عو امل خطوره مؤثرة

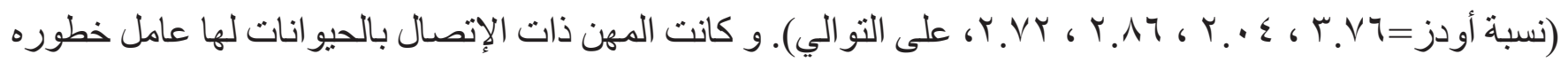

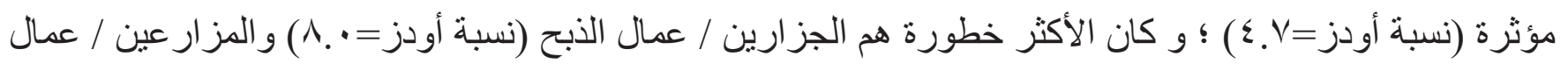

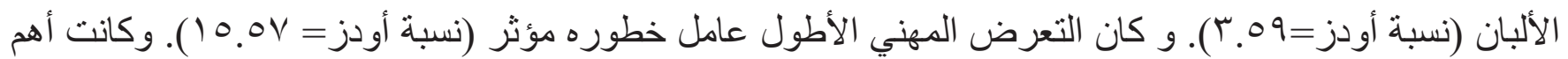
أعر اض المرض عند التشخيص هى الحمى و الإضطر ابات العضلية الهيكلية. وكانت أهم العلامات الرئيسية هى إرتفاع درجة الحر ارة وتضخم الكبد والطحال. وكان إختبار التر اص القياسى (SAT) عيار / / • بس هى نقطة الفصل للتشخيص


إيجابية في ؟ ـه٪ من الحالات مع عدم وجود فروق ذات دلالة إحصائية بين إختبار التر اص القياسى و إيجابية مزر عة الدم. وكانت الأجسام المضادة الموجبة من النوع م (IgM) و ج (IgG) و المكتشفه بو اسطة إختبار الإليزا (ELISA)

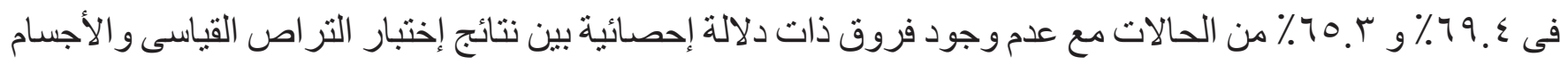

المضادة الموجبة من النوع م (IgM) و ج (IgG). الإستنتاجات : داء البروسيلا البشرى لديه العديد من عوامل الخطوره التي يمكن الوقاية منها و يعتمد تشخيصه بشكل أساسى على وجود عو امل الخطورهو الإشتباه سريريًا و إختبار التراص القياسى (SAT) عيار = أو أكبر 